\section{Les trypanosomes africains ont le sens du sacrifice}

Étienne Pays
Laboratoire de parasitologie moléculaire, Institut de biologie et de médecine moléculaire (IBMM), Université Libre de Bruxelles (ULB),

12, rue des Professeurs Jeener et Brachet, B604l Gosselies, Belgique.

epays@ulb.ac.be

\section{Infection par le trypanosome : rôle du système immunitaire et de la glycoprotéine VSG}

Les trypanosomes africains, dont le prototype s'appelle Trypanosoma brucei, sont des protozoaires flagellés capables d'infecter de nombreux mammifères suite à leur inoculation intraveineuse lors d'un repas sanguin pris par des mouches infectées, les Glossines, appelées communément mouches tsétsé. Chez I'homme, cette infection provoque la maladie du sommeil. Elle affecte aussi les bovins, compromettant l'élevage du bétail sur un tiers du continent africain. Les trypanosomes sont bien connus pour leur capacité extraordinaire à jongler avec le système immunitaire de leur hôte, non seulement par l'amoindrissement de la qualité de la réponse immune (perte de la spécificité et de la mémoire immunitaires), mais aussi et surtout, par un pouvoir illimité de variation antigénique [1]. Ces deux propriétés sont dues à une glycoprotéine attachée à la membrane plasmique du parasite par un radical d'ancrage de type glycosyl-phosphatidyl-inositol (GPI) : la glycoprotéine VSG (variant surface glycoprotein). La VSG est présente en quantité extrêmement abondante sous la forme d'un manteau protecteur dense et homogène. Lors de chaque vague de prolifération du parasite, atteinte en quelques jours, cette glycoprotéine est libérée par le clivage de l'ancre GPI, active les macrophages et perturbe le système immunitaire. Parallèlement, un changement d'expression génique conduit à la production de variants de VSG. Collectivement, ces phénomènes expliquent que le trypanosome provoque des infections chroniques de longue durée, ce qui est nécessaire pour assurer la transmission du parasite d'un hôte à l'autre lors d'improbables piqûres par la mouche tsétsé.

\section{L'adénylate cyclase du trypanosome :} son rôle au stade initial de l'infection parasitaire

\section{Adénylate cyclase et stress environnemental}

Bien que des informations très nombreuses et détaillées aient été obtenues concernant les mécanismes mis en œuvre par le trypanosome pour manipuler le système immunitaire au cours de l'infection, rien n'était connu des stratégies d'invasion du parasite, lorsque les premiers parasites envahisseurs sont confrontés à un système immunitaire parfaitement fonctionnel.

Mon laboratoire s'intéressait depuis longtemps à la fonction de l'adénylate cyclase du trypanosome et au rôle de l'AMP cyclique (AMPC) dans l'infection parasitaire. D'une part, nous avions observé que la synthèse d'AMPc était pratiquement indétectable chez le parasite dans des conditions normales de croissance, et ce messager n'avait pas été impliqué non plus dans les nombreuses tentatives d'identification des voies de signalisation intracellulaire fonctionnant chez le parasite. D'autre part, de façon assez paradoxale, le génome de Trypanosoma brucei était caractérisé par une expansion considérable du nombre de gènes codant pour l'adénylate cyclase, avec presque 100 copies codant pour différentes isoformes. Un indice important nous a mis sur la piste et permis de résoudre ce paradoxe : nous avons observé que diverses conditions de stress environnemental provoquaient une

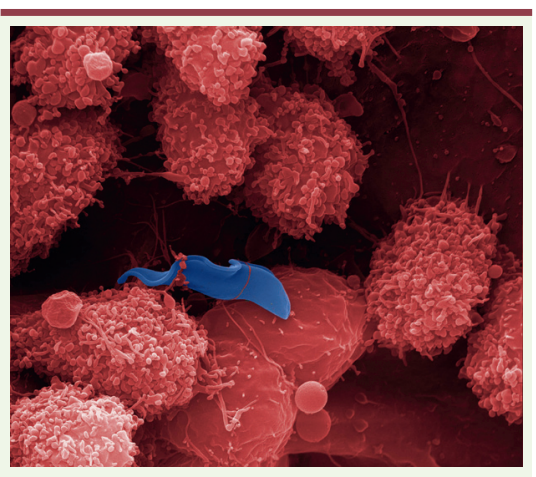

Figure 1. Trypanosoma brucei au milieu des cellules myéloïdes hépatiques lors d'une infection chez la souris. Au moment de sa phagocytose par des cellules myéloïdes du foie (en rose), le trypanosome (en bleu) synthétise de I'AMPc pour inhiber la production de la cytokine antiparasitaire TNF- $\alpha$. L'image est obtenue par microscopie électronique à balayage de sections de foie de souris infectées par Trypanosoma brucei (๔ G. Vanwalleghem, D. Monteyne et D. Pérez-Morga, Université Libre de Bruxelles et Centre pour microscopie et imagerie moléculaires, Gosselies, Belgique).

activation considérable de l'adénylate cyclase, l'effet optimal étant observé lors de la lyse cellulaire [2]. Par ailleurs, le séquençage du génome de différents trypanosomatidés a révélé que la multiplication des gènes d'adénylate cyclase était caractéristique des parasites à vie extracellulaire, présents dans la circulation sanguine ( $T$. congolense, $T$. vivax), et donc en contact constant avec les cellules du système immunitaire; à l'opposé, le nombre de copies de gènes codant les adénylate cyclases était moindre chez les parasites essentiellement intracellulaires (T. cruzi, Leishmania), donc moins exposés à ces cellules immunitaires. Ainsi, la fonction des isoformes d'adénylate cyclase du 


\section{Adénylate cyclases de Trypanosoma brucei}
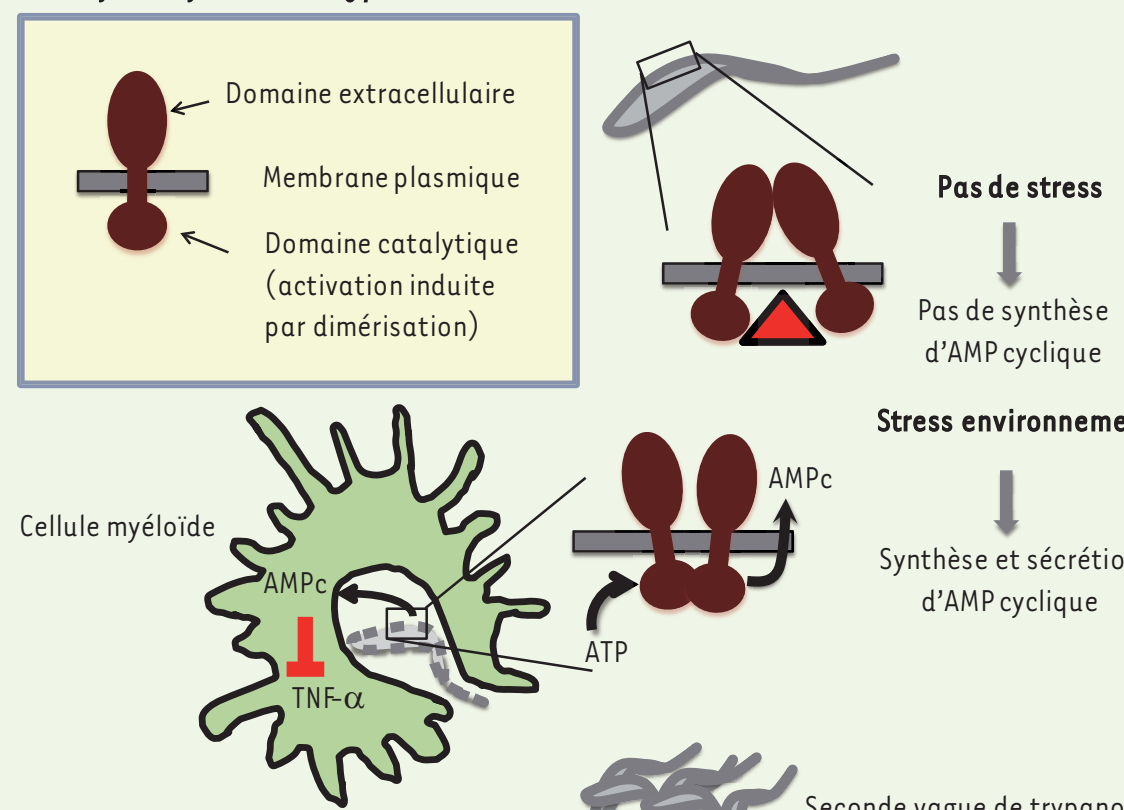

Premiers envahisseurs:

mort par stress de la phagocytose

et action du TNF- $\alpha$

du foie (Figure 1) était nécessaire pour inhiber la production de la cytokine TNF- $\alpha$ (tumor necrosis factor- $\alpha$ ) par ces mêmes cellules, via l'activation de la protéine kinase $A$ (PKA, dont l'activité est dépendante du niveau d'AMPc) (Figure 2). Le TNF- $\alpha$ étant fortement impliqué dans la réaction antiparasitaire en tout début d'infection, la stimulation des cyclases provoquée par la capture et la mort des premiers trypanosomes permet, en neutralisant cette réponse cytokinique de l'hôte, aux autres parasites de proliférer. Donc, la mort des «premiers 》 trypanosomes envahisseurs permet à leurs congenères d'établir l'infection (Figure 2).

Que l'adénylate cyclase puisse être associée à l'inhibition de la réponse immune par des pathogènes était déjà connu: les bactéries Bordetella nosomes vivants de développer l'infection.

trypanosome pouvait être liée au stress du parasite provoqué par l'interaction des cellules immunitaires avec le pathogène sanguin.

\section{Le sacrifice des premiers envahisseurs}

Afin de tester cette hypothèse, nous avons tiré parti d'une propriété intrinsèque de l'activité des enzymes adénylate cyclase du trypanosome: la dimérisation du site catalytique. Chez les trypanosomatidés, les différentes formes d'adénylate cyclase possèdent une structure qui s'apparente à celle des récepteurs transmembranaires avec un seul domaine catalytique intracellulaire; par conséquent, ces enzymes doivent dimériser pour s'acti- ver. Nous avons construit des trypanosomes transgéniques surexprimant une forme «dominante négative » du domaine catalytique: celui-ci a été inactivé par mutagenèse dirigée de manière à réduire l'activité de l'adénylate cyclase globale une fois le dimère formé (effet dominant négatif). De façon très claire, une diminution de l'activité de l'adénylate cyclase a été observée, et celle-ci était associée à une forte réduction de la capacité du parasite à résister à l'immunité innée de l'hôte en tout début d'infection [3]. Plus précisément, nous avons pu montrer que l'activation des adénylate cyclases liée à la phagocytose du parasite par les cellules myéloïdes pertussis et Brucella anthracis libèrent des toxines à activité adénylate cyclase qui empoisonnent et détruisent les macrophages [4]. Ce qui est original dans le cas des trypanosomes, c'est le sacrifice, au bénéfice de la population, de certains parasites intoxiquant les macrophages de l'intérieur au cours de leur capture lors de l'étape initiale de l'infection. Une autre observation intéressante concerne le contrôle de l'activité des adénylate cyclases: elles sont maintenues sous forme inactive de façon permanente dans les conditions normales d'approvisionnement énergétique, et leur activation spontanée en conditions de stress est un exemple remarquable d'adaptation immédiate d'un organisme à un environnement 
modifié en l'absence de tout apport énergétique. Un tel mécanisme d'activation, particulièrement adapté pour un parasite confronté aux changements soudains de son milieu, vaut aussi pour l'activation de l'enzyme qui clive la structure d'ancrage GPI de la VSG en conditions de stress [2].

\section{Conclusion et perspectives}

Le rôle des adénylate cyclases à l'interface hôte-parasite explique l'expansion et le polymorphisme de cette famille enzymatique chez les trypanosomes: en multipliant les isoformes, le parasite évite d'être reconnu de façon spécifique et efficace par le système immunitaire de l'hôte. Une telle diversification a déjà été observée pour d'autres enzymes parasitaires exposées au système immunitaire: par exemple les trans-sialidases de T. cruzi ou les kinases de Toxoplasma $[5,6]$.
Enfin, la fonction des adénylates cyclases, qui cible les cellules infectées de l'hôte plutôt qu'une fonction du parasite lui-même explique, d'une part, les échecs des tentatives d'identification des ligands capables d'activer ces enzymes, et d'autre part, l'absence de voie de signalisation intracellulaire dépendant de l'AMPc chez ces parasites. $\varepsilon$ matière de lutte contre ce parasite, bien qu'il soit difficile dans l'immédiat d'identifier des perspectives concrètes, la conception de stratégies empêchant la dimérisation des adénylates cyclases et, ainsi, bloquant les étapes initiales de colonisation de l'hôte par le parasite pourrait se révéler prometteuse. $\diamond$ African trypanosomes have the sense of sacrifice

\section{LIENS D'INTÉREิT}

L'auteur déclare n'avoir aucun lien d'intérêt concernant les données publiées dans cet article.

\section{RÉFÉRENCES}

1. Pays $\varepsilon$, Vanhamme L, Pérez-Morga D. Antigenic variation in Trypanosoma brucei : facts, challenges and mysteries. Curr Opin Microbiol $2004 ; 4$ : 369-74.

2. Rolin S, Hanocq-Quertier J, Paturiaux-Hanocq F, et al. Simultaneous but independent activation of adenylate cyclase and glycosylphosphatidylinositolphospholipase $\mathrm{C}$ under stress conditions in Trypanosoma brucei.J Biol Chem 1996 ; 271 : 10844-52.

3. Salmon D, Vanwalleghem G, Morias Y, et al. Adenylate cyclases of Trypanosoma brucei inhibit the innate immune response of the host. Science 2012 ; 337 : 463-6.

4. McDonough KA, Rodriguez A. The myriad roles of cyclic AMP in microbial pathogens : from signal to sword. Nat Rev Microbiol $2012 ; 10: 27-38$.

5. Ratier L, Urrutia M, Paris G, et al. Relevance of the diversity among members of the Trypanosoma cruzi trans-sialidase family analyzed with camelids singledomain antibodies. PLoS One 2008 ; 3 : e3524.

6. Saeij JPJ, Boyle JP, Coller S, et al. Polymorphic secreted kinases are key virulence factors in Toxoplasmosis. Science 2006 ; 314 : 1780-3.

\section{NOUVELLE}

\section{Une micropuce de diagnostic hybride pour la détection d'ARN non codants}

Konstantinia Skreka ${ }^{1}$, Mathieu Rederstorff ${ }^{1,2}$

\author{
${ }^{1}$ Innsbruck biocenter, département de génomique et \\ RNomique, faculté de médecine, Innrain, 6020 Innsbruck, \\ Autriche ; \\ ${ }^{2}$ Université de Lorraine, Biopôle, CNRS UMR 7214 AREMS \\ (ARN-RNP, structure-fonction-maturation, enzymologie \\ moléculaire et structurale), 9, avenue de la forêt de Haye, \\ Vandœuvre-lès-Nancy, 54506, France. \\ mathieu.rederstorff@maem.uhp-nancy.fr
}

> Malgré l'importance majeure des ARN messagers (ARNm), les transcriptomes eucaryotes sont essentiellement constitués d'ARN non messagers ou ARN non codants (ARNnc) pour des protéines. Ces derniers sont impliqués dans des processus biologiques variés mettant en jeu des mécanismes moléculaires et cellulaires multiples. Récemment, les ARNnc ont même été décrits comme d'excellents marqueurs diagnostiques pour de nombreuses pathologies, notamment en cancérologie [1].

\section{Séquençage à haut débit ou} micropuces: le débat fait rage

Pour la réalisation d'une analyse transcriptomique, les approches basées sur le séquençage à haut débit tendent à remplacer progressivement les approches basées sur l'utilisation de micropuces. $\varepsilon n$ effet, ces dernières nécessitent la connaissance a priori des séquences à étudier. Elles sont opaques à l'identification de nouvelles molécules, telles que des nouveaux variants issus de l'épissage alternatif d'un préARNm ou des nouveaux petits ARNnc, contrairement aux approches par séquençage dites « ouvertes ». Les approches par séquençage sont, en revanche, encore peu utilisées en diagnostic [2, 3]. Récemment, plusieurs études ont été entreprises avec comme objectif la comparaison de l'efficacité, de la sensibilité et de la reproductibilité de ces deux approches majeures de transcriptomique. L'analyse d'échantillons biologiques ou d'une population synthétique contrôlée d'ARN a permis de comparer les résultats obtenus par séquençage ou micropuce. Bien que les deux approches apparaissent très reproductibles et spécifiques, les approches basées sur l'utilisation de puces demeurent sensiblement plus précises en termes de quantification absolue d'espèces très peu abondantes [4, $5]$.

Malheureusement, les conditions optimisées d'analyse par micropuce d'ARNm ne peuvent pas s'appliquer à l'étude des 